\title{
Fault diagnosis for supporting rollers of the rotary kiln using the dynamic model and empirical mode decomposition
}

\author{
Kai Zheng*, Yun Zhang**, Chen Zhao***, Tianliang Li**** \\ *School of Mechanical and Electronic Engineering, Wuhan University of Technology, Wuhan 430070, China, \\ E-mail: zhengkai2001@163.com \\ **School of Mechanical and Electronic Engineering, Wuhan University of Technology, Wuhan 430070, China, \\ E-mail:whkasco@aliyun.com \\ ***School of Mechanical and Electronic Engineering, Wuhan University of Technology, Wuhan 430070, China, \\ E-mail: zhaochen@whut.edu.cn \\ ****School of Mechanical and Electronic Engineering, Wuhan University of Technology, Wuhan 430070, China, \\ E-mail: tianliangliwhut@sina.com
}

cross $^{\text {ref }}$ http://dx.doi.org/10.5755/j01.mech.22.3.13072

\section{Introduction}

Rotary kiln is a typical large slow-speed running (2-6 rpm) mechanical equipment. As key equipment, rotary kiln is widely used in the cement industry, metallurgical industry and environmental protection industry. It is mainly composed by transmission system, driving system, supporting rollers and heat exchange system [1], as shown in Fig. 1. The plain bearings in the supporting rollers enable the rotary kiln to run at a low speed with heavy load (12 supporting rollers support $15,000-20,000 \mathrm{kN}$ load) [1-2]. And the operation state of the rotary kiln was largely determined by the working condition of supporting rollers [1-3]. During long-term operation, supporting rollers vibrate due to the kiln crank [2]. Early fault diagnosis for the rotary kiln's supporting rollers has important engineering significance in that it can help to reduce equipment maintenance cost and economic loss resulting from production suspension of the rotary kiln [1-2]. To achieve the above-mentioned purposes, it is of great importance to study the dynamic model and identify the fault features of the supporting rollers.

Until now, a limited research has been done for the fault diagnosis of the supporting rollers. Eng. Zbignie et al have studies the causes of the kiln crank, and pointed that the kiln crank would affect the supporting bearings and lead to the deflection of the supporting rollers' shaft [4]. Świtalski, Maciej proposed a method for the diagnostic of the rotary kiln's technical state by the measurement of the shell's elastic [6]. Alma Žiga Hertz, et al. studied the distribution of contact pressure between the supporting roller and tyre based on Hertz contact theory and did a simulation research based on finite element method [7]. Gebhart, Walter et al proposed the measurement principle and method of the supporting roller's deflection. They adopted curve fitting method to calculate the deflection of the roller shaft [3]. X.j. Li, et al. presented that dynamic change of the operating axis of the rotary kiln will lead to complex vibration of the equipment, so as to speed up fatigue failure of the supporting rollers. They used transfer matrix method to establish a kinetic model of the rotary kiln's cylinder [8]. Stamboliska Zhaklina, Eugeniusz Rusiński, et al. studied the cause of the vibration of the rotary kiln's supporting rollers and pointed out that the vibration of the supporting rollers essentially resulted from the kiln crank of rotary kiln's cylinder. Meanwhile, they made an in-depth study on the fault mode of the supporting rollers and proposed an online signal processing method based on the FFT method [1-2].

However, few work has been involved the vibration mechanism and the fault extraction of the supporting rollers. In order to explore the impact of the crank of the rotary kiln's cylinder on the supporting rollers as well make fault diagnosis, this paper put forward the dynamic model of the supporting rollers and made a numerical simulation analysis. Moreover, as the vibration signals of the supporting rollers are normally characterized with nonstationary behaviour, to analyze the vibration signals with nonstationary properties, we presented features extraction and fault diagnosis method of the rotary kiln supporting rollers based on empirical mode decomposition (EMD), so as to realize the condition monitoring of the low-speed operation rotary kiln.

The remainder of this paper is organized as follows. In Section 2, an analysis was made on the impact of the rotary kiln crank on the supporting rollers and the dynamic model was established. In Section 3, the numerical simulation analysis was done. In Section 4, a features extraction and fault diagnosis method based on empirical mode decomposition was presented. In Section 5, an analysis was made on the vibration signals from the industry field experiment based on the numerical simulation analysis result and the proposed the fault diagnosis method. Section 6 is a conclusion of this paper.

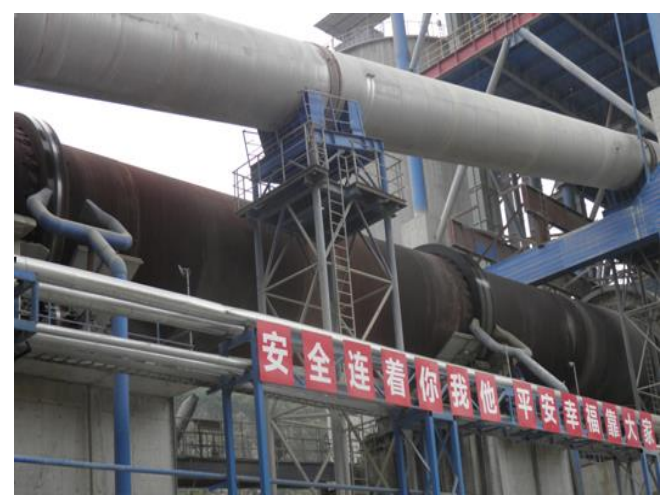

Fig. 1 Rotary kiln in a cement plant of China 


\section{Physical model of the supporting rollers}

\subsection{The effect to supporting rollers of kiln operation}

According to FMECA result, the fault of the supporting rollers is a main factor which can lead to the breakdown of the rotary kiln [1-2]. According to [3], the fault of the supporting rollers is mainly caused by the kiln crank generated by the internal thermal process of the rotary kiln. During long-term operation, the profile of the cylinder will change, which will lead to misalignment between the geometric center and the rotation center, thereby causing the eccentricity of the cylinder section. And the dynamic load caused by the deformation of the cylinder eccentricity gives rise to the vibration of the supporting roller. The snowball effect in the rotary kiln's cylinder will further intensify the vibration of the supporting rollers. According to [1], the materials will form a basic ball in the kiln called the snowball effect during the operation of the rotary kiln. The ball moves slowly around the kiln's axis. When the weight of the snowball exceeds the adhesive force on the kiln coating and the snowball surface, the snowball will come off from the kiln coating. Under extreme conditions, such a thermal effect may lead to significant load unbalance of the supporting rollers.

Fig. 2 shows the straightness deviation of the rotary kiln we measured in a cement plant in China. It can be found that the straightness deviation of rotary kiln cylinder was large due to various factors. The supporting rollers in the three stations of the rotary kiln are free from the restraint of the tyre. When the snowball effect appears in the rotary kiln's cylinder, the straightness deviation of the axis will increase, leading to heavy cyclic loads, which will cause the vibration of supporting rollers in the radial direction, as shown in Fig. 3. If the vibration amplitude is too large, the plain bearing may not make up the displacement of the roller shaft, so as to cause frictional heating in them. And the bearing alloy will melt with the high temperature, causing significant failure of the supporting rollers.

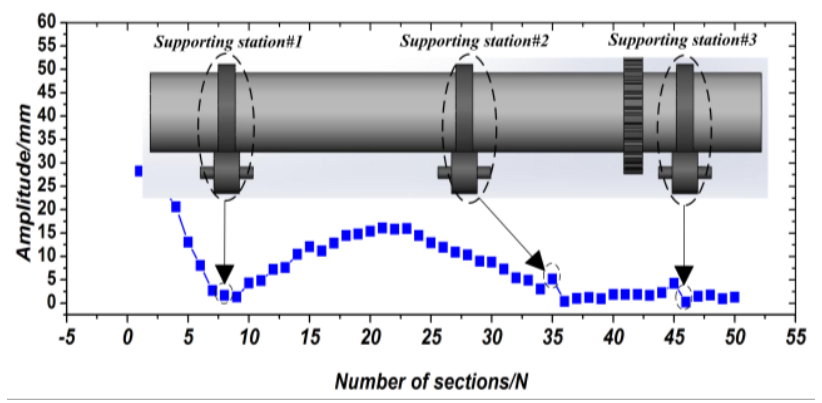

Fig. 2 The measurement result of straightness deviation of the rotary kiln in a cement plant

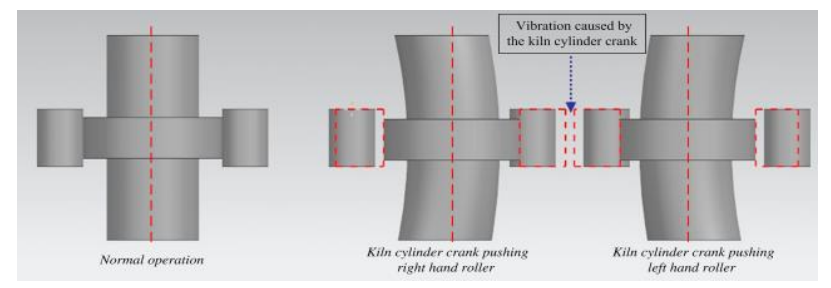

Fig. 3 The vibration of supporting rollers caused by the Kiln crank effect
To establish the dynamic equation of the supporting rollers, it is very important to describe the cyclic load resulting from the kiln crank.According to [2,4], the change of the kiln cylinder profile is an important factor resulting in the dynamic load, and the eccentricity (e) can be used as a main parameter for assessing the section profile. To establish the dynamic model of the supporting rollers, a simplified formula was put forward for the cyclic load calculation. And it can be expressed as:

$$
f=m_{1} \omega_{1}^{2} e_{1}
$$

Where $m_{1}$ is the equivalent unbalance mass at the corresponding station, $\omega_{1}$ is the kiln cylinder's rotational speed and $e_{1}$ is the eccentricity of the cylinder's cross section.

\subsection{The equations of the supporting rollers}

To establish the dynamic model of the low-speed rotary kiln's supporting system under cyclic load, we established a simplified model as shown in Fig. 4. In Fig. 4, supposing that the rotary kiln's supporting roller is a disc with a mass $m$, the supporting shaft is an isotropic shaft without mass, and the left and right rollers at the same position of the rotary kiln bear the same load, and that $O X Y$ is the inertial coordinate system, $O x y$ is the rotating coordinate system, $O$ is the whirling center (rotation center) of the supporting roller, $O^{\prime}$ is the centroid, $R$ is the radius of the supporting roller, $e$ is the eccentricity of the center section of the supporting roller. We can derive the equation of the supporting roller's vibration according to Lagrange's dynamical equations.

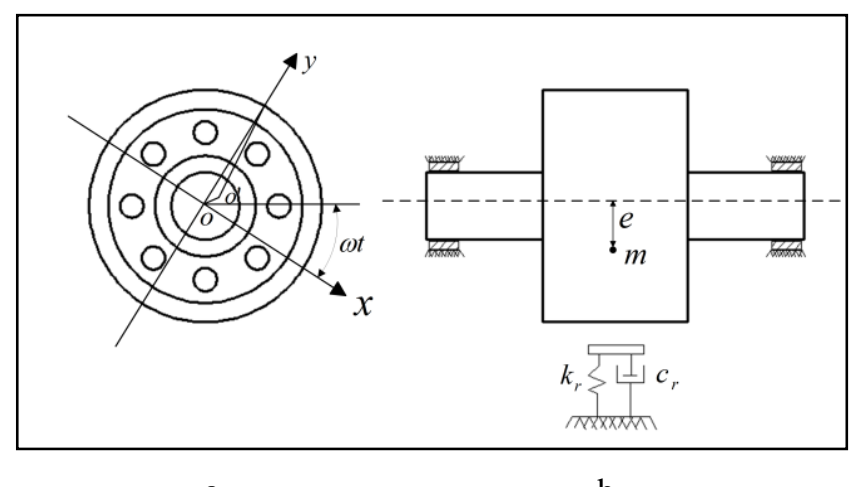

a

b

Fig. 4 The model schematic of supporting rollers: a - side view; b - front view

According to the force analysis for the supporting rollers, the external loads of them can be expressed by Eqs. (2):

$$
\left[\begin{array}{c}
F_{j}^{(n)} \\
Q_{j}^{(n)}
\end{array}\right]=\left[\begin{array}{l}
m e \omega^{2} \cos (\omega t+\beta) \\
m e \omega^{2} \cos (\omega t+\beta)
\end{array}\right]+\left[\begin{array}{l}
f \cos \left(\omega_{1} t\right) \\
f \sin \left(\omega_{1} t\right)
\end{array}\right],
$$

where $f$ is the cyclic load caused by the kiln crank, $\omega$ is the rotation speed of the supporting roller and $e$ is the eccentricity of the supporting roller. Based on Lagrange's dynamical equations and formulas (2) we can derive the dynamic equation of the supporting roller as expressed by Eq. (3): 


$$
\left[\begin{array}{cc}
m & 0 \\
0 & m
\end{array}\right]\left[\begin{array}{l}
\ddot{x} \\
\ddot{y}
\end{array}\right]+\left[\begin{array}{cc}
c_{1} & 0 \\
0 & c_{2}
\end{array}\right]\left[\begin{array}{l}
\dot{x} \\
\dot{y}
\end{array}\right]+\left[\begin{array}{cc}
k_{1} & 0 \\
0 & k_{2}
\end{array}\right]\left[\begin{array}{l}
x \\
y
\end{array}\right]=\left[\begin{array}{l}
F_{j}^{(n)} \\
Q_{j}^{(n)}
\end{array}\right],
$$

where $k_{1}$ and $k_{2}$ are the stiffness values of the supporting roller in $x$ and $y$ directions, $c_{1}$ and $c_{2}$ are the damping forces of the supporting roller in $x$ and $y$ directions, respectively; as we suppose the supporting roller is isotropous, it can be regarded that $k_{1}=k_{2}, c_{1}=c_{2} ; m$ is the equivalent mass of the supporting roller.

\section{Numerical prediction for the response of supporting rollers}

\subsection{The simulation parameters estimation}

It is great important to carry out a careful estimation of the physical parameter values of the supporting rollers to perform a numerical simulation. However, it is difficult to take this task as that the specific parameters of the support rollers depend on the production, the length and the tyre number of a rotary kiln. In this research, we take a qualitative analysis to the numerical simulation of a three tryes rotary kiln with the production of 5000-6500 $t / d$. According to [9], the basic material of the supporting rollers is ZG42GrMo and the elasticity modulus of it is $2.09 \times 10^{5} \mathrm{MPa}$. The mass density of the rollers is $7.86 \times 10^{-6} \mathrm{~kg} / \mathrm{mm}^{3}$ and the radius is $1150 \mathrm{~mm}$. Based on the above data, we can obtain the approximate physical parameter values of supporting rollers, which are shown in Table 1

Table 1

Estimation parameters of the supporting rollers used for numerical simulations

\begin{tabular}{|c|c|}
\hline Parameter & Value \\
\hline Rollers stiffness of $x, y$ axis $k_{1}, k_{2}$ & $1.48 \times 10^{5} \mathrm{~N} / \mathrm{m}$ \\
\hline $\begin{array}{c}\text { Rollers viscous damping of } x, y \\
\text { axis } c_{1}, c_{2}\end{array}$ & $6.92 \times 10^{4} \mathrm{Ns} / \mathrm{m}$ \\
\hline Equivalent mass of rollers $m$ & $4.05 \times 10^{4} \mathrm{~kg}$ \\
\hline $\begin{array}{c}\text { Equivalent imbalance mass of } \\
\text { station\#2 } m_{1}\end{array}$ & $7.97 \times 10^{5} \mathrm{~kg}$ \\
\hline The rotating speed of the rollers $\omega$ & $10.7 \mathrm{r} / \mathrm{min}$ \\
\hline The rotating speed of the kiln $\omega_{1}$ & $4 \mathrm{r} / \mathrm{min}$ \\
\hline Mass unbalance angle $\beta$ & $0 \mathrm{rad}$ \\
\hline
\end{tabular}

\subsection{Numerical analysis of the supporting rollers}

In this section, the dynamic response of the supporting rollers under the influence of the kiln cylinder crank was studies. The influence of the cyclic load to supporting rollers caused by thermal effects of the kiln cylinder was stimulated.

As it is difficult to achieve analytical solution of the complex dynamics equation, the numerical methods was used to solve the problem. And the fourth-order Runge-Kutta method [10] is selected to solve the Eq. (10) to obtain the dynamics response of the supporting rollers.

The effect on the supporting rollers of the rotary kiln crank was analyzed in this section. According to section II, during the operation of the kiln, it will change the dimensional size as the result of the internal thermal pro- cesses. As the rotation center and the center of mass is not overlap in the cylinder section, thus producing section eccentricity. Therefore, the eccentricity of the cross section can be used as a main parameter to represent the kiln crank. And we compared the vibration response of the supporting roller under whether or not there is kiln crank. And the simulation result was shown in Fig. 5. Furthermore, the relationship between the section eccentricity of the kiln cylinder and the amplitude of the kiln harmonic and the amplitude of the rollers harmonic was analyzed, and the result was shown in Fig. 6.

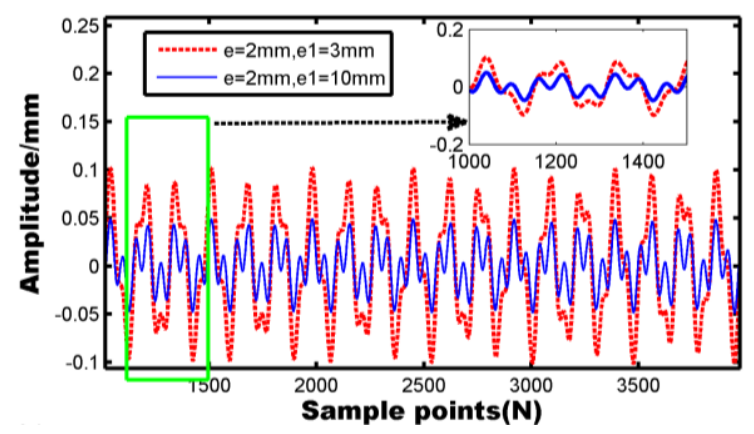

a

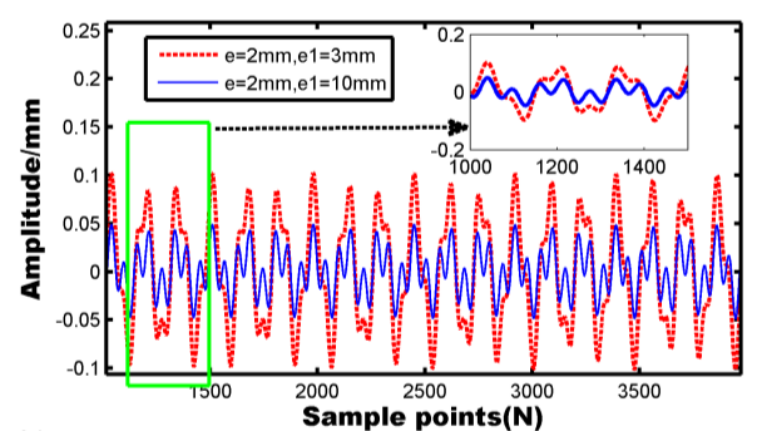

b

Fig. 5 The vibration response in the $y$ direction of the supporting rollers when $e_{1}=3$ and $e_{1}=10 \mathrm{~mm}$ : a - time domain; $\mathrm{b}$ - frequency domain

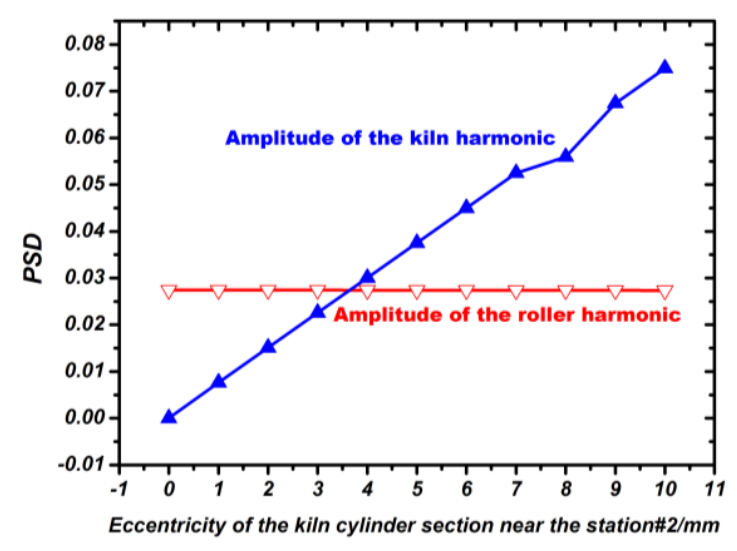

Fig. 6 Relationship between the section eccentricity of the kiln cylinder and the amplitude of the kiln harmonic and the amplitude of the rollers harmonic

From the above numerical simulation results, it can be found that:

1) when there is kiln crank, there will have a kiln harmonics $(\mathrm{KH})$ in the vibration signal;

2) when the magnitude of the kiln harmonic 
$(\mathrm{KH})$ increased, it is a sign that the crank of the rotary kiln is being enhanced. And it has larger deviations from the normal operation in terms of eccentricity and deflections from regular rotation axis in section corresponding to monitored station;

3) the frequency of kiln harmonic is consistent with rotary kiln rotating speed while the frequency of rollers harmonic is equal to the supporting rollers rotation speed. Therefore, the operation condition of the rotary kiln can be monitored by collecting the vibration signals of the supporting rollers.

\section{Feature extraction using empirical mode decomposition method}

As mentioned in section 3, the vibration signals of the supporting rollers contain multiple fault information. According to the numerical analysis for the supporting rollers, it can be found that the vibration signal mainly includes the harmonic component caused by the kiln crank and the harmonic component caused by the deflection of the roller shaft. In [17], authors pointed out that it also contains the harmonic component of the surface characteristic, such as the harmonic component of waviness, the roughness and micro-irregularities. In fact, the vibration signal are nonlinear, non-stationary signal which are not suitable processed by the traditional signal processing method such as fast Fourier transform (FFT) or Wavelet transform (WT) [12-14]. In this research, we proposed a method for feature extraction of supporting rollers based on empirical mode decomposition.

\subsection{Empirical mode decomposition}

Empirical mode decomposition was first proposed as a part of Hilbert-Huang transform (HHT) by Norden E.huang, which is effective method to analysis the nonlinear, non-stationary signal [13]. As the key part of HHT, the method of EMD to decompose signal is intuitive, direct and adaptive. This decomposition method is based on local characteristic of local time domain of signals. Based on this characteristic, any linear, stationary or nonlinear, non-stationary signal can be decomposed into a set of Intrinsic Mode Functions (IMFs) which are amplitude and frequency modulated signals. It has been proven to be an effective method in analyzing nonstationary signals for rotational machine fault detection $[15,16]$. According to $[15,16]$, each IMF satisfies two basic conditions:

1) over the entire dataset, the number of extreme and the number of zero crossings must either be equal or differ at most by one;

2) at any time point, the local mean value of the envelope which defined by the average of the maximum and minimum envelopes is zero.

The specific processes of EMD are described as follows:

Step 1. For the given signal $x(t)$, construct its upper envelope $e_{\max }(t)$ and lower envelope $e_{\min }(t)$ by connecting all local maxima and local minima with cubic spline functions. And:

$$
m_{11}=\frac{e_{\max }(t)+e_{\min }(t)}{2} .
$$

Step 2. Compute the envelopes mean $m_{11}$, and $x(t)-m_{11}=h_{1}(t)$. And the definition of IMF is proposed mainly to get the physical meaning of the instantaneous frequency.

Step 3. If $h_{1}(t)$ satisfies the definition of IMF, then we can obtain the first-order IMF $I M F_{1}=h_{1}(t)$. And then go to the next step. In addition, the IMF component $c_{1}(t)=h_{1 k}(t)$ is saved. If it is not the IMF, repeat Steps 1-3. The stop condition for the iteration is given by:

$$
S D=\sum_{t=0}^{T}\left[\frac{\left|h_{i(j-1)}(t)-h_{i j}(t)\right|^{2}}{h_{i(j-1)}^{2}(t)}\right],
$$

where $h_{i(j-1)}(t)$ and $h_{i j}(t)$ denote the IMF candidates of the $j-1$ and $j$ iterations, respectively, and usually, SD is set between 0.2 and 0.3 .

Step 4. Separate $c_{1}(t)$ from $x(t)$, we could get $r_{1}(t)=x(t)-c_{1}(t), r_{1}(t)$ is treated as the original data and repeat the above processes, the second IMF component $c_{2}(t)$ of $x(t)$ could be got. Let us repeat the process as described above for $\mathrm{n}$ times, then $\mathrm{n}$-IMFs of signal $x(t)$ could be got.

Step 5. The decomposition process can be stopped when $r_{(n)}(t)$ becomes a monotonic function from which no more IMF can be extracted. We can finally obtain:

$$
x(t)=\sum_{i=1}^{n} c_{i}(t)+r_{n} .
$$

Residue $r_{(n)}(t)$ is the mean trend of $x(t)$. The IMFs $c_{1}(t), c_{2}(t), \ldots, c_{\mathrm{n}}(t)$ include different frequency bands ranging from high to low. The frequency components contained in each frequency band are different and they change with the variation of signal $x(t)$, while $r_{(n)}(t)$ represents the central tendency of signal $x(t)$.

\subsection{Feature extraction and fault diagnosis procedure}

Numerical simulation results shown that the kiln harmonic $(\mathrm{KH})$ and the rollers harmonic $(\mathrm{RH})$ will lead to significant change as the kiln cylinder crank happens or the rollers behavior changes. Therefore, the energy variation of the kiln harmonic $(\mathrm{KH})$ and the rollers harmonic $(\mathrm{RH})$ of the vibration signals can reflect the running condition of the rotary kiln. When the malfunction occur in a mechanical equipment, the energy of the vibration signal would change strongly in some frequency bands, but in other frequency bands the energy maybe change weakly [12]. According to [16], the IMF energy moment not only contains the size of IMFs energy, but also considers the distribution of IMF's energy change with the time parameter $t$. It can be used to express the energy distribution of the fault characteristic frequency component for the vibration signals. In this research, the IMF's energy moment is employed for fault diagnosis of the supporting rollers. And the procedures are summarized as follows:

Step 1. The signal $x(t)$ is decomposed into several IMFs based on the EMD method, and the redundant IMFs were removed based on the correlation coefficient. And the physical meaning of the effective IMFs can be found.

Step 2. The HHT marginal spectrum of each IMF component was calculated based on Hilbert transformation. And the IMF energy moment was calculated. The operation state of the rotary kiln was determined according to 
the IMF energy moment. According to [16], the energy of each IMF component can be calculated by the Eq. (7):

$$
E_{i}=\sum_{j=1}^{N}(k \Delta t)\left|c_{i}(k \Delta t)\right|^{2}
$$

where $\Delta t$ is the period of data samples, $N$ is the total number of data samples, and $k$ represents the number of data samples. Eq. (8):

The IMF energy moment can be calculated by

$$
\boldsymbol{T}=\left\{E_{1}, E_{2} \ldots E_{i} \ldots E_{n}\right\} / \sum_{i=1}^{n} E_{i},
$$

where $\sum_{i=1}^{n} E_{i}$ is the summary energy of all the IMF components of the signal, $n$ is the number of the effective IMF components. $\mathbf{T}$ is the percent of the energy of IMF components in the whole signal energy.

\section{Experiment and discussions}

The measurement system comprises an acquisition card, the non-contact eddy current sensors, the hall sensor and the measuring software, as shown in Fig. 7, b. During the measurement, the non-contact eddy current sensor is mounted through fixture to keep the sensor probe in the contact direction of roller and kiln tyre ( $y$ direction), as shown in Fig. 7, a, and probe should be kept close enough to the surface of the roller according to the eddy current sensor range.

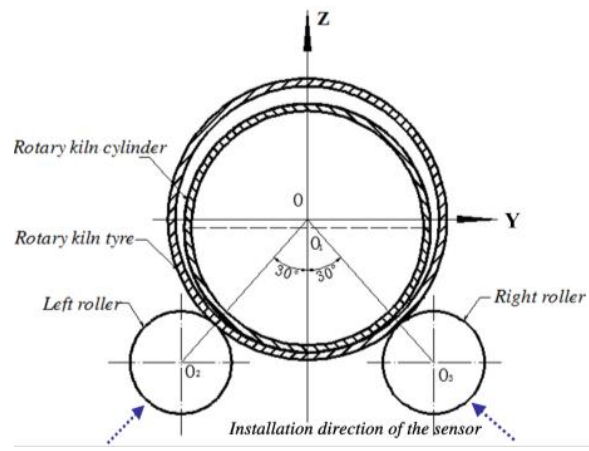

$\mathrm{a}$

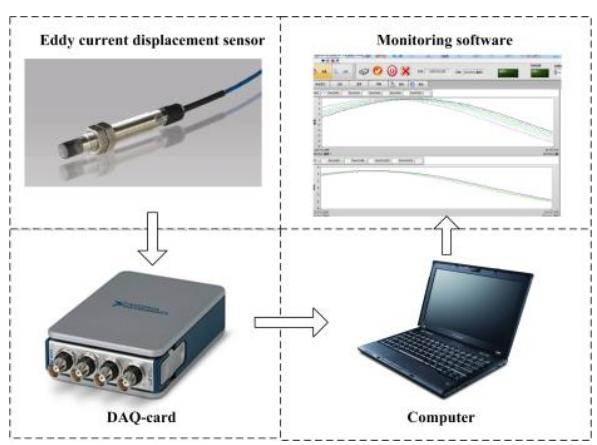

b

Fig. $7 \mathrm{a}$ - The installation position (in the $y$ direction) of the displacement sensors and $\mathrm{b}$ - schematic diagram of the experimental setup

The hall sensor is installed in a fixed position of rotary kiln to interact with magnet mounted on the kiln cylinder generating pulse signal per lap which is used to synchronize the collected vibration signal and the rotation of the rotary kiln. Then the collected vibration signals are sent by NI data acquisition card to the upper computer for management and storage by Labview-based data acquisition software and SQL database.

We did several experiments in cement plants in China, and the measured object was a rotary kiln which consist three tyres. The speed of the kiln cylinder was $3.5 \mathrm{r} / \mathrm{min}$ while the supporting rollers were $10.5 \mathrm{r} / \mathrm{min}$. The vibration signals of all the supporting rollers were collected based on the measurement system. The sample frequency of the channel was settled to $20 \mathrm{HZ}$.

The kiln crank leading to the vibration of the supporting rollers often takes place in the second and third rollers. Therefore, for verifying the dynamics model of the supporting rollers, the vibration signal of the third right supporting rollers was used to analyze, as shown in Fig. 8. Also the profile data of the cross section near to the third station were collected. The geometric centre, base circle and eccentricity can be calculated by the computational algorithm proposed by literature $[4,5]$.

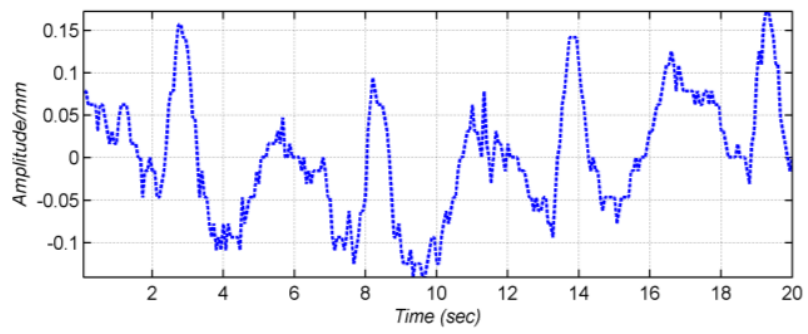

Fig. 8 Vibration signal of the third right roller
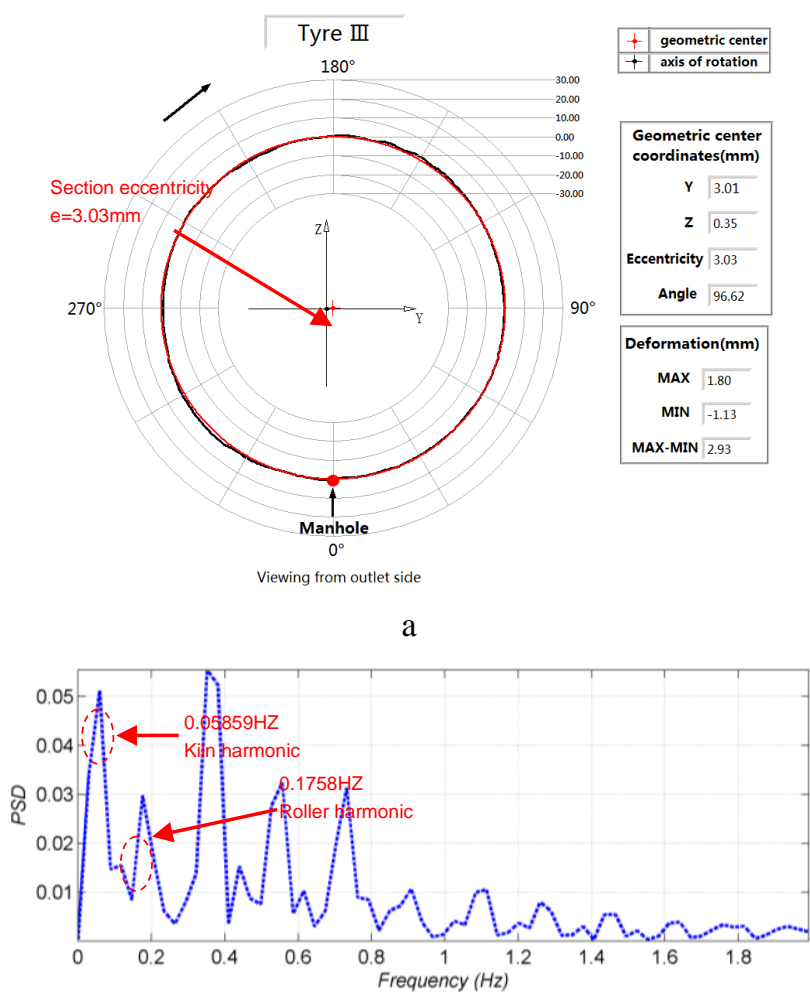

b

Fig. 9 a - The calculation result of the section eccentricity near to the third station; $b$ - the spectrum of the vibration signal of third right supporting roller 
Based on the computational algorithm, the eccentricity of the cross section near to the third station is $e=3.03 \mathrm{~mm}$, as shown in Fig. 9, a. It means that there should be $\mathrm{RH}$ and $\mathrm{KH}$ components in the vibration signals of the supporting rollers according to the numerical simulation result. We adopted FFT method to process the vibration signals of the third right roller, as shown in Fig. 9, b. And we can find two major frequency components $(0.05859 \mathrm{HZ}$ and $0.1758 \mathrm{HZ})$ in the signals, which are basically the same as the rotation frequencies of the rotary kiln's cylinder $(0.5833 \mathrm{HZ})$ and supporting roller $(0.175 \mathrm{HZ})$, respectively. Eccentricity appeared in the cylinder's section and correspondingly, KH component appeared in the vibration signals of the supporting roller. This was the same as the simulation result and proved that the model was correct. And the physical map of in field industry experiment was shown in Fig. 10.
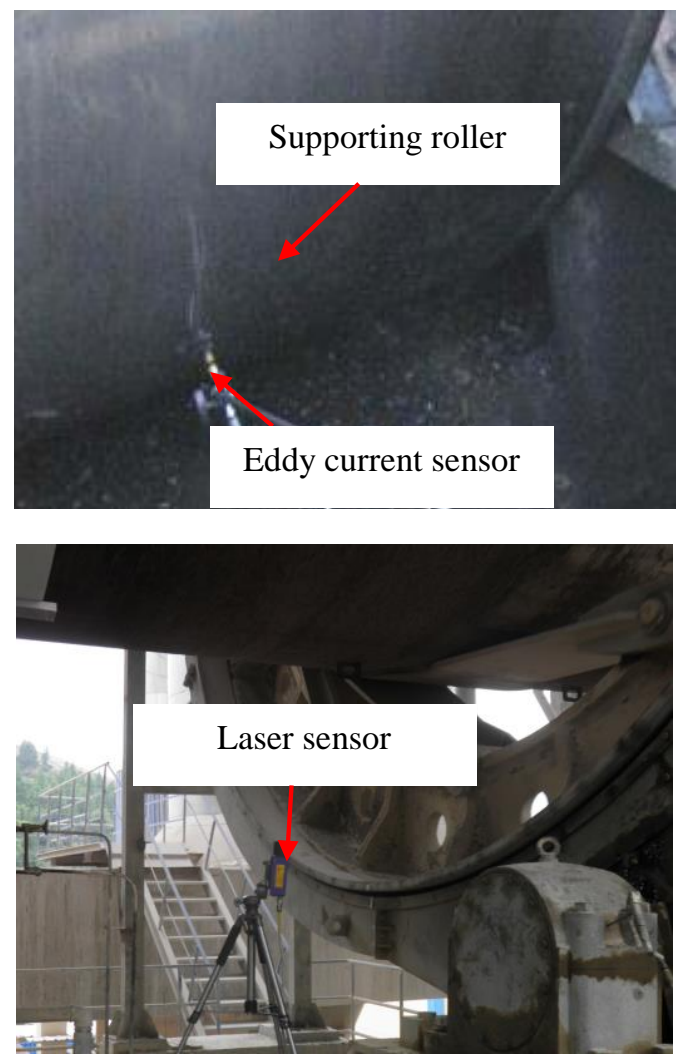

Fig. 10 Physical map of experiment for verifying the dynamics model of the supporting rollers

We processed the vibration data of all supporting rollers based on empirical mode decomposition method. And the vibration signal of the third right supporting roller was analyzed. EMD processing results are shown in Fig. 11. After the vibration signals were processed with EMD method, the IMF components ranked from high to low frequency. In particular, the specific physical meanings of each IMF component are as follows: IMF5 (frequency: $0.05859 \mathrm{HZ}$ ) is harmonic component caused by the kiln crank; IMF4 (frequency: $0.1758 \mathrm{HZ}$ ) is the vibration component caused by the supporting roller shaft deflection; according to $[17,18]$, IMF3 is the ripple deformation component of the supporting roller's surface; IMF2 is the harmonic component of the supporting roller's surface roughness; IMF1 is the harmonic component of microscopic deformation.
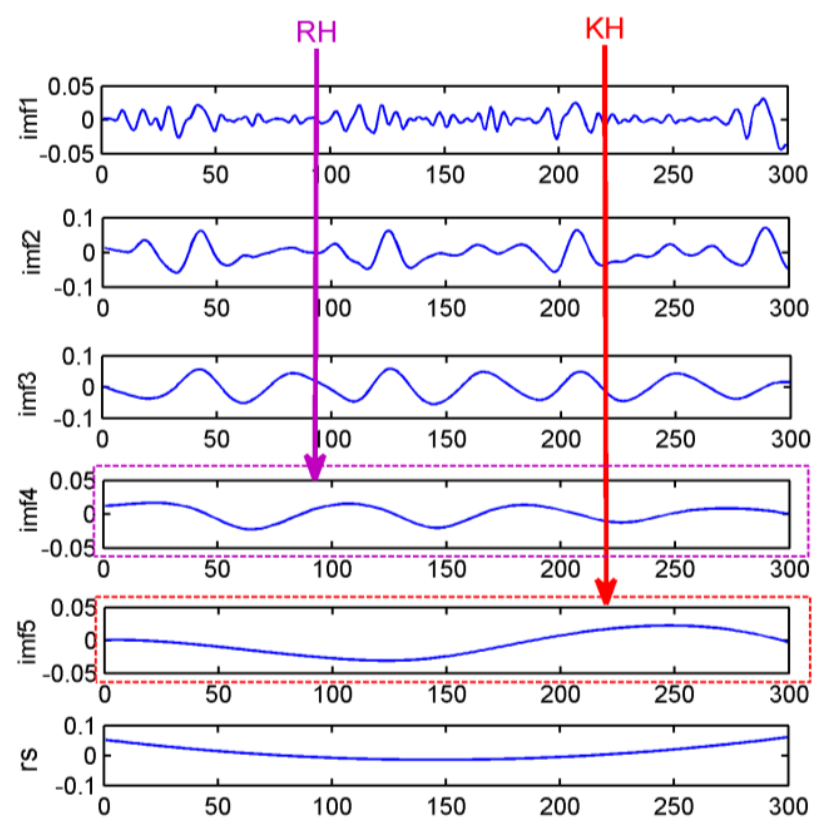

Fig. 11 The EMD decomposition result of the vibration signal of the third right supporting roller

In order to decide the operation status of the rotary kiln, the IMF energy moment is proposed according to the definition mentioned in section 4 . And the proposed IMF energy moment is $\boldsymbol{T}=\left[\begin{array}{lll}\alpha & \beta & \lambda\end{array}\right]$, whose computation formulas is shown in (9):

$$
\alpha=E_{5} / E ; \beta=E_{4} / E ; \lambda=\left(E_{3}+E_{2}+E_{1}\right) / E,
$$

where $\alpha$ represents the energy coefficient of the harmonic component caused by kiln crank; $\beta$ represents the energy coefficient of the harmonic component resulting from deflection of the supporting roller's shaft; and $\lambda$ represents the energy coefficient of harmonic component of the supporting roller's surface roughness and harmonic.

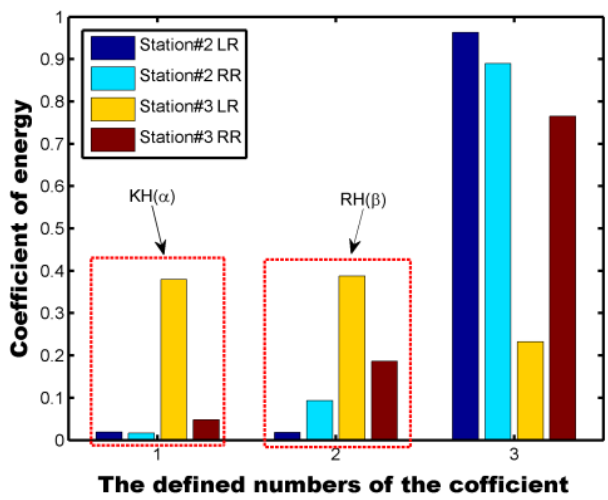

Fig. 11 Calculation results of IMF energy coefficient of the supporting rollers

From the calculation result, we can find that the energy coefficient of IMF1, IMF2 and IMF3 components ( $\lambda$ ) representing the contour deformation of the second left and right supporting rollers and the third right supporting roller are great, and the energy coefficient of IMF4 $(\beta)$ and IMF5 $(\alpha)$ components representing the kiln crank and deflection of the supporting roller's are relative small. And the energy coefficient of IMF4 and IMF5 of the second left 
supporting roller are great, as shown in Fig 12. It shows that the contour of the second left and right supporting rollers and the third right supporting roller deformed to some extent due to heavy load on them, but the impact vibration resulting from the kiln's thermal effect is small. However, the impact vibration of the third left supporting roller is big due to the heavy kiln crank. If no maintenance measures are taken, the bearing of the supporting roller's bearing may burn out and even melt due to the high temperature.

\section{Conclusion}

The operation condition of the rotary kiln can be reflected by monitoring the vibration signals of the supporting rollers as they are core components of it. In order to extract the feature of the vibration signals, the numerical simulation of the supporting rollers was analyzed and a signal processed method based on EMD was proposed. From the above simulation and experimental results, the contributions and conclusion of this research are made as follows:

1) The numerical simulation result indicated that when there is kiln crank, there will have a kiln harmonics $(\mathrm{RH})$ in the vibration signal. When the kiln crank enhance, the amplitude of kiln harmonics $(\mathrm{RH})$ will increases while the rollers harmonics (RH) almost has no change.

2) The changes of the kiln harmonics and rollers harmonics reflect the energy variation of vibration signals when the rotary kiln is in different running statuses. Therefore, a fault diagnosis method based on EMD was proposed. The signal was decomposed into a serial of IMF components based on EMD. An analysis was made over energy distribution of fault features under different frequency bands, and the IMF energy moment was calculated to determine the operating state of the rotary kiln.

3) The simulation and experimental results indicated that the proposed method could be used to extract the feature for the vibration signals of the supporting rollers effectively, whichprovide a new method for the fault diagnosis of the low speed machinery like the rotary kiln.

\section{References}

1. Rusinski, E.; Stamboliska, Z.; Moczko, P. 2013. Proactive control system of condition of low-speed cement machinery, Automation in Construction 31: 313-324. http://dx.doi.org/10.1016/j.autcon.2012.12.001.

2. Stamboliska, Z.; Rusiński, E.; Moczko, P. 2015. Proactive Condition Monitoring of Low-Speed Machines. Springer International Publishing, $186 \mathrm{p}$. http://dx.doi.org/10.1007/978-3-319-10494-2_5.

3. Gebhart, W.M.; Stutz, T. 2007. Process knowhow-routine support roller shaft deflection measurements-surprising results, ZKG International 60(7): 50-55.

4. Krystowczyk, Z. 2004. Geometry measurements of kiln shell in dynamic conditions, Cement \& Building Materials 16: 34-37.

5. Kai Zheng; Yun Zhang; Chen Zhao; Lei Liu 2015. Rotary kiln cylinder deformation measurement and feature extraction based on EMD method, Engineering Letters 23(4): 283-291.
6. Świtalski, M. 2010. The measurement of shell's elastic ovality as essential element of diagnostic of rotary drum's technical state, Diagnostyka 1(53): 37-47.

7. Žiga, A.; Karač, A.; Vukojević, D. 2013. Analytical and numerical stress analysis of the rotary kiln ring, Tehnički Vjesnik 20(6): 941-946.

8. Li, X.; Shen, Y.; Wang, S. 2011. Dynamic modeling and analysis of the large-scale rotary machine with multi-supporting, Shock and Vibration 18(1-2): 53-62. http://dx.doi.org/10.3233/SAV-2010-0573.

9. Xuchang Jiang. 2012. Adjustment of the supporting rollers for the rotary kiln, China building material press.

10. Guo, C.; Al-Shudeifat, M.A.; Yan, J.; Bergman, L.A.; McFarland, D.M.; Butcher, E.A. 2013. Application of empirical mode decomposition to a Jeffcott rotor with a breathing crack, Journal of Sound and Vibration 332(16): 3881-3892.

http://dx.doi.org/10.1016/j.jsv.2013.02.031.

11. Gao, Q.; Duan, C.; Fan, H.; Meng, Q. 2008. Rotating machine fault diagnosis using empirical mode decomposition, Mechanical Systems and Signal Processing 22(5): 1072-1081.

http://dx.doi.org/10.1016/j.ymssp.2007.10.003.

12. Liu, B.; Riemenschneider, S.; Xu, Y. 2006. Gearbox fault diagnosis using empirical mode decomposition and Hilbert spectrum, Mechanical Systems and Signal Processing 20(3): 718-734. http://dx.doi.org/10.1016/j.ymssp.2005.02.003.

13. Huang, N.E.; Shen, Z.; Long, S.R.; Wu, M.C.; Shih, H.H.; Zheng, Q.; ... Liu, H.H. 1998. The empirical mode decomposition and the Hilbert spectrum for nonlinear and non-stationary time series analysis, In Proceedings of the Royal Society of London A: Mathematical, Physical and Engineering Sciences 454(1971): 903-995. The Royal Society. http://dx.doi.org/10.1098/rspa.1998.0193.

14. He, D.; Li, R.; Zhu, J. 2013. Plastic bearing fault diagnosis based on a two-step data mining approach, IEEE Transactions on Industrial Electronics 60(8): 3429-3440. http://dx.doi.org/10.1109/TIE.2012.2192894.

15. Li, R.; He, D. 2012. Rotational machine health monitoring and fault detection using EMD-based acoustic emission feature quantification, IEEE Transactions on Instrumentation and Measurement 61(4): 990-1001. http://dx.doi.org/10.1109/TIM.2011.2179819.

16. Bin, G.F.; Gao, J.J.; Li, X.J.; Dhillon, B.S. 2012. Early fault diagnosis of rotating machinery based on wavelet packets-Empirical mode decomposition feature extraction and neural network, Mechanical Systems and Signal Processing 27: 696-711. http://dx.doi.doi.org/10.1016/j.ymssp.2011.08.002.

17. Xia, C.; Wu, Y.; Lu, Q.; Ju, B. 2014. Surface characteristic profile extraction based on Hilbert-Huang transform, Measurement 47: 306-313. http://dx.doi.doi.org/10.1016/j.measurement.2013.08.0 66.

18. Muralikrishnan, B.; Raja, J. 2008. Computational Surface and Roundness Metrology, Springer Science \& Business Media, 263 p.

http://dx.doi.org/10.1007/978-1-84800-297-5. 
Kai Zheng, Yun Zhang, Chen Zhao, Tianliang Li

FAULT DIAGNOSIS FOR SUPPORTING ROLLERS OF THE ROTARY KILN USING THE DYNAMIC MODEL AND EMPIRICAL MODE DECOMPOSITION

\section{S u m m a r y}

Rotary kiln is key equipment widely used in the cement, metallurgical and environmental protection industry. The operation state of the rotary kiln was largely determined by the working condition of supporting rollers. This paper models the vibration mechanism of the supporting rollers under the rotary kiln crank caused by the internal thermal process. Based on the numerical analysis, a methodology based on empirical mode decomposition (EMD) for the fault diagnosis of the supporting rollers was proposed. By using EMD, the complicated vibration signal of the kiln can be decomposed into a number of intrinsic mode functions (IMFs). An analysis was made over the energy moment of IMF components to indicate the energy variation of the kiln harmonic and the roller harmonic of the vibration signals. Simulation and in field experiment results proved that the proposed method provided a validity of the approach for the condition monitoring and fault diagnosis of the low-speed machinery like the rotary kiln.

Keywords: Supporting rollers, fault diagnosis, numerical analysis, empirical mode decomposition, vibration monitoring.

Received September 08, 2015 Accepted May 11, 2016 